\title{
Grand Challenges in Physical Sensors
}

\author{
Joel Villatoro ${ }^{1,2 *}$ \\ ${ }^{1}$ Department of Communications Engineering, University of the Basque Country UPV/EHU, Bilbao, Spain, ${ }^{2} I K E R B A S Q U E$, \\ Basque Foundation for Science, Bilbao, Spain
}

Keywords: optical fiber sensing, mechanical sensing, electronic sensing devices, optical sensors and sensing, physical sensors

\section{OPEN ACCESS}

Edited by:

Dermot Diamond,

Dublin City University, Ireland

Reviewed by:

Jian Zhang,

Hefei University of Technology, China

*Correspondence:

Joel Villatoro

agustinjoel.villatoro@ehu.eus

Specialty section:

This article was submitted to

Physical Sensors,

a section of the journal

Frontiers in Sensors

Received: 06 April 2020

Accepted: 22 May 2020

Published: 30 June 2020

Citation:

Villatoro J (2020) Grand Challenges in

Physical Sensors. Front. Sens. 1:1.

doi: 10.3389/fsens.2020.00001
Since ancient times the monitoring of physical parameters like temperature, wind velocity, atmospheric pressure, tilt, and force, among others, has been important for humanity. The first sensors or devices to measure such parameters were bulky. In addition, they were inaccurate, as the user or observer was in charge of reading and interpreting the sensor signal. Nonetheless, the data gathered with such devices were useful for navigation and astronomy.

Nowadays, our society is more industrialized, and we use, and to some extent depend on, a myriad of sophisticated devices, machines, and instruments. The performance and functionalities of such devices, machines, and instruments depend indirectly on the diverse high-precision sensors installed within them. Therefore, there has been an increasing need for more and more sensors. Such sensors are used to monitor parameters as diverse as liquid level, flow, temperature, pressure, magnetic fields, acoustic signals, distance, proximity, inclination, rotation, touch, motion, light intensity, acceleration, color, and many others. Presently, sensors are being used to monitor physical parameters even in the most hostile or challenging of environments, such as, for example, force and ultrasound inside the human body, acoustic waves in the deep sea, pressure in turbines and oil wells, and rotation and acceleration in space.

Sensors can provide data and information on multiple physical parameters that are crucial for controlling diverse industrial processes or that are vital in medical applications, meteorology, or scientific research. That is the reason why these sensors have become ubiquitous. Sensors provide useful data on the environment where they are located, which is vital for the performance and functionality of devices and machines. They also provide continuous information on the status or health of structures, materials, or devices wherein they are embedded. Thus, they are placed at multiple points to collect as much data as possible on diverse physical parameters. In this manner, sensors make materials, devices, or structures, smart, more efficient or functional, and safer.

In most cases, sensors only provide data on a single parameter, the one they are conceived for, but in more recent applications, two or more sensors work together. For example, accelerometers and gyroscopes in unmanned aircraft provide more information to the aircraft itself, such as position, altitude, distance traveled, etc. Accelerometers and gyroscopes in wearable devices or in sporting equipment work together to provide information as diverse as distance traveled by a person, the number of impacts, the power and location of the impact, etc. All this information helps athletes to improve their performance.

Presently, it is impossible to conceive critical infrastructures, vehicles, instruments, cities, homes, gadgets, etc., without multiple physical sensors. The mission of such sensors is to provide real-time data on a number of parameters ranging from temperature, acceleration, and sound, to tilt or beats of a person's heart. Thanks to sensors, critical infrastructures such as pipelines, roads, buildings, instruments, vehicles, etc., have "artificial nerves" or senses. Modern medical instruments and surgical robots, for example, include several sensors that monitor not only parameters of medical relevance but also physical parameters like force or pressure that provide haptic feedback. Another example is self-driving cars, in which a massive number of 
parameters are constantly monitored to make them more secure and cleaner. A third example includes the sensors in smartphones and watches. Such sensors provide data as diverse as a person's heart rate, the status of the battery, the orientation of the screen, proximity to an object, or the intensity of the ambient light.

The general trend to make everything smarter, safer, or cleaner, as well as the trend to add more functionalities to instruments, devices, gadgets, smartphones, appliances, etc., has increased the demand for monitoring more parameters in diverse circumstances. This, in turn, has increased the types of sensors and the technologies used to fabricate them. In general, electronic sensors are preferred in a wide variety of applications, as they have small dimensions, have low weight, and are reliable and cost-effective. However, electronic sensors may not work properly or may not be appropriate or recommended for certain applications. In some situations, optical sensors are the only viable solution, as they are insensitive to electromagnetic interference (Du et al., 2020). Modern endoscopes, for example, include fiber optic force and acoustic sensors and monitor such parameters inside the human body. Optical fibers are also used to monitor strain, temperature, and vibrations (Fernández-Ruiz et al., 2020) at millions of points in critical infrastructures such as pipelines, power lines, perimeters, and borders.

So, the obvious question is: what are the grand challenges for physical sensors in the years to come? There is no simple answer to this question, as the applications of sensors are diverse, and many different types of sensors exist.

Let us start with the challenges from the scientific point of view. The frontiers of physical sensors regarding sensitivity, resolution, measuring range, precision, accuracy, etc., are expanded almost every day. Undoubtedly, nano and quantum technologies (Dowran et al., 2018; Mitchell and Alvarez, 2020) will contribute to pushing the limits of physical and other sensors. By using single or entangled photons or other quantum resources the sensitivity of optical and electronic sensors can be improved beyond the so-called shot-noise limit. Therefore, in the near future, it will be possible to develop sensors capable of detecting parameters with precision and resolution that today's sensors cannot achieve. The enhancement of sensitivity with squeezed states of light was demonstrated in the gravitational wave detector VIRGO (Acernese et al., 2019). Similar concepts can be used in the development of other highly sensitive quantum sensors. New nanomaterials or effects that appear at the nanoscale can also be used to improve the sensitivity of a sensor or to develop completely new, ultra-miniature sensors.

From the technological point of view, the challenge for sensors is to reduce their cost, size, or energy consumption substantially. Fiber optic sensors, for example, are much more expensive than electronic ones. Hence, to reduce the cost of fiber sensors, it is crucial to investigate new approaches to devising such sensors, their components, or the ways they are interrogated. Wearable devices, on the other hand, have limited use due to the short duration of the charge of batteries. Therefore, it would be interesting to investigate new mechanisms for powering sensors
(Huang et al., 2020), for example, with the heat or the movement of the human body or with the parameter to be monitored. In autonomous cars, sensors must be less bulky and less expensive; in addition, they must be highly reliable and precise. Moreover, they must respond quickly. Sensors with such attributes will contribute to developing cleaner, more reliable, and affordable self-driving cars.

The processing and interpretation of the data and signals generated by sensors also present important challenges, and this is an interesting area of research in sensor technology. Currently, sensors are used to monitor multiple physical parameters in real time, and this will not change much in the future. In many industrial, scientific, and other applications, sensors can provide massive amounts of data that have to be collected, stored, processed, and interpreted at high speed to provide information in real time. In these cases, concepts and approaches used in artificial intelligence and deep learning (Zhang et al., 2019) and techniques used in big data (Teh et al., 2020) are and will increasingly be taken into account.

We now live in the era of the Internet of Things (IoT), where the biggest challenge for physical (and any other) sensors will be to provide data, at literally every instant, on the parameter they are conceived to monitor as well as to communicate the sensed data to other devices and to learn from the data (Masoud et al., 2019; Muthu et al., 2020). Therefore, in the coming years, we will see sensors that play roles beyond sensing.

The recent global pandemic caused by the coronavirus has presented significant challenges for physical sensors to solve urgent societal needs, for example, temperature sensors to rapidly identify people with fever (Su et al., 2019) in airports and bus/train stations. In this case, the challenge is to measure body temperature without touching people, rapidly, with high precision and, ideally, measuring it for several people simultaneously. Flow and pressure sensors incorporated into breathing aids are also critically important for helping infected people to breathe more easily; hence, such sensors help save lives in intensive care units. On the other hand, smart doors and taps help to avoid propagating the virus, as proximity sensors installed in them can open such doors or taps without touching them. In the near future, we will see how several sensors installed in smartphones work together to help to combat the spread of the coronavirus. Smartphones will provide us with a sophisticated proximity detection system to alert us on possible exposure to infected people.

Physical and other sensors will continue to provide artificial senses and more functionalities to all sorts of things and make them smarter, safer, cleaner, and more friendly for the user. During the recent global pandemic, it is evident that physical sensors are also important and necessary and even can help save lives.

\section{AUTHOR CONTRIBUTIONS}

The author confirms being the sole contributor of this work and has approved it for publication. 


\section{REFERENCES}

Acernese, F., Agathos, M., Aiello, L., Allocca, A., Amato, A., Ansoldi, S., et al. (2019). Increasing the astrophysical reach of the Advanced Virgo detector via the application of squeezed vacuum states of light. Phys. Rev. Lett. 123:231108. doi: 10.1103/PhysRevLett.123.231108

Dowran, M., Kumar, A., Lawrie, B., Pooser, R., and Marino, A. (2018). Quantum-enhanced plasmonic sensing. Optica 5, 628-633. doi: 10.1364/OPTICA.5.000628

Du, C., Dutta, S., Kurup, P., Yu, T., and Wang, X. (2020). A review of railway infrastructure monitoring using fiber optic sensors. Sens. Actuators A 303:111728. doi: 10.1016/j.sna.2019.111728

Fernández-Ruiz, M. R., Soto, M. A., Williams, E. F., Martin-Lopez, S., Zhan, Z., Gonzalez-Herraez, M., et al. (2020). Distributed acoustic sensing for seismic activity monitoring. APL Photonics 5:030901. doi: 10.1063/1.5139602

Huang, L., Lin, S., Xu, Z., Zhou, H., Duan, J., Hu, B., et al. (2020). Fiber-Based energy conversion devices for human-body energy harvesting. Adv. Mater. 32:1902034. doi: 10.1002/adma.201902034

Masoud, M., Jaradat, Y., Manasrah, A., and Jannoud, I. (2019). Sensors of smart devices in the internet of everything (IoE) era: big opportunities and massive doubts. J. Sens. 2019:6514520. doi: 10.1155/2019/6514520

Mitchell, M. W., and Alvarez, S. P. (2020). Colloquium: quantum limits to the energy resolution of magnetic field sensors. Rev. Mod. Phys. 92:021001. doi: 10.1103/RevModPhys.92.021001
Muthu, B., Sivaparthipan, C. B., Manogaran, G., Sundarasekar, R., Kadry, S. Shanthini, A., et al. (2020). "IOT based wearable sensor for diseases prediction and symptom analysis in healthcare sector," in Peer-to-Peer Networking and Applications. 1-12. doi: 10.1007/s12083-019-00823-2

Su, C. P., Wang, Y. Y., Ku, K. C., and Fang, C. T. (2019). Clinical and epidemiological characteristics of imported dengue fever among inbound passengers: Infrared thermometer-based active surveillance at an international airport. PLoS ONE 14:e0225840. doi: 10.1371/journal.pone.0225840

Teh, H. Y., Kempa-Liehr, A. W., and Wang, K. I. (2020). Sensor data quality: a systematic review. J. Big Data 7:11. doi: 10.1186/s40537-020-0285-1

Zhang, C., Patras, P., and Haddadi, H. (2019). Deep learning in mobile and wireless networking: a survey. IEEE Commun. Surveys Tutorials 21, 2224-2287. doi: 10.1109/COMST.2019.2904897

Conflict of Interest: The author declares that the research was conducted in the absence of any commercial or financial relationships that could be construed as a potential conflict of interest.

Copyright $\odot 2020$ Villatoro. This is an open-access article distributed under the terms of the Creative Commons Attribution License (CC BY). The use, distribution or reproduction in other forums is permitted, provided the original author(s) and the copyright owner(s) are credited and that the original publication in this journal is cited, in accordance with accepted academic practice. No use, distribution or reproduction is permitted which does not comply with these terms. 